\title{
Rigidity of Complete Gradient Shrinkers with Pointwise Pinching Riemannian Curvature
}

\author{
Yawei Chu $\mathbb{D},{ }^{1}$ Dehe Li $\mathbb{D},{ }^{2}$ and Jundong Zhou $\mathbb{D}^{1}$ \\ ${ }^{1}$ School of Mathematics and Statistics, Fuyang Normal University, Fuyang, Anhui 236037, China \\ ${ }^{2}$ School of Mathematics and Statistics, Anyang Normal University, Anyang, Henan 455000, China \\ Correspondence should be addressed to Dehe Li; lidehehe@163.com
}

Received 1 July 2021; Accepted 4 August 2021; Published 25 August 2021

Academic Editor: Shyamal Kumar Hui

Copyright (c) 2021 Yawei Chu et al. This is an open access article distributed under the Creative Commons Attribution License, which permits unrestricted use, distribution, and reproduction in any medium, provided the original work is properly cited.

Let $\left(M^{n}, g, f\right)$ be a complete gradient shrinking Ricci soliton of dimension $n \geq 3$. In this paper, we study the rigidity of $\left(M^{n}, g, f\right)$ with pointwise pinching curvature and obtain some rigidity results. In particular, we prove that every $n$-dimensional gradient shrinking Ricci soliton $\left(M^{n}, g, f\right)$ is isometric to $\mathbb{R}^{n}$ or a finite quotient of $\mathbb{S}^{n}$ under some pointwise pinching curvature condition. The arguments mainly rely on algebraic curvature estimates and several analysis tools on $\left(M^{n}, g, f\right)$, such as the property of $f$-parabolic and a Liouville type theorem.

\section{Introduction}

An $n$-dimensional $(n \geq 3)$ Riemannian manifold $\left(M^{n}, g\right)$ is called a Ricci soliton if there exist a smooth vector field $X$ and a constant $\lambda \in \mathbb{R}$ on $M^{n}$ such that

$$
R c+\frac{1}{2} \mathscr{L}_{X} g=\lambda g
$$

where $R c$ and $\mathscr{L}_{X} g$ denote the Ricci tensor and the Lie derivative of $g$ in the direction of $X$, respectively, and $\lambda$ is sometimes called the soliton constant. The soliton is shrinking, steady, or expanding if $\lambda>0, \lambda=0$, or $\lambda<0$, respectively. When $X$ is a gradient of a smooth function $f$ on $M^{n}$, the soliton is called a gradient Ricci soliton and (1) becomes

$$
R c+\text { Hess } f=\lambda g
$$

Note that when $X$ or $\nabla f$ is a Killing vector field, equations (1) and (2) reduce to the Einstein equation. Thus, Ricci solitons are natural generalizations of Einstein maifolds. In particular, when $X=0$ or $f$ is a constant, the soliton is trivial.

In recent decades, increasing investigations have been done to the rigidity of gradient shrinking Ricci solitons (gradient shrinker for short). In dimension 2, Hamilton [1] showed that a gradient shrinker is isometric to $\mathbb{R}^{2}$ or to a quotient of $\mathbb{S}^{2}$. The first rigidity theorem in dimension 3 was proved by Ivey [2] saying that a 3-dimensional compact gradient shrinker is a quotient of $\mathbb{S}^{3}$. In the noncompact case, the revelent rigidity result was showed by Perelman [3] with noncollapsing assumption, which was removed by Naber [4] later. Adopting different arguments, Ni and Wallach [5] and Cao et al. [6] obtained the full classification; they proved that any 3-dimensional gradient shrinker must be isometric to $\mathbb{R}^{3}$ or to a quotient of $\mathbb{R} \times \mathbb{S}^{2}$ or $\mathbb{S}^{3}$. Some relevant conclusions can be found in $[4,7,8]$.

When $n \geq 4$, under the assumption of nonnegative curvature operator or vanishing Weyl tensor, Naber [4], Ni and Wallach [5], Petersen and Wylie [8], and Zhang [9] proved corresponding rigidity results on gradient shrinkers, which were improved by Catino [10] using a general pointwise pinching condition on the Weyl tensor.

On the other hand, Munteanu and Wang [11] investigated the curvature behavior of 4-dimensional gradient shrinker and proved that there exists a constant $C>0$ for 4-dimensional gradient shrinkers with bounded scalar curvature $R$ so that

$$
|R m| \leq C R,
$$


which along with the fact $|R m|^{2}=\left|R^{\circ} m\right|^{2}+\left(R^{2} / 6\right)$ implies

$$
|\stackrel{R}{R} m| \leq\left(C^{2}-\frac{1}{6}\right) R^{2},
$$

and $C \geq(\sqrt{6} / 6)$. Here, $R^{\circ} m$ is the trace-free curvature tensor.

In [12], the authors established the following rigidity theorem under pointwise pinching condition of $R^{\circ} m$ :

Theorem 1 (Theorem 1.1 in [12]). Let $\left(M^{n}, g, f\right)$ be an $n$ -dimensional $(n \geq 3)$ complete gradient shrinker. If

$$
|\stackrel{\circ}{R} m| \leq \frac{1}{C(n)}\left(\lambda-\frac{n-2}{n(n-1)} R\right),
$$

then $\left(M^{n}, g\right)$ is isometric to $\mathbb{R}^{n}$ or a finite quotient of $\mathbb{S}^{n}$.

In this paper, we will restrict our attention to the rigidity of gradient shrinkers with pointwise pinched conditions associated with $R^{\circ} m$ and the traceless Ricci tensor $R^{\circ} c=R c-(R / n) g$. By establishing $f$-parabolic and algebraic curvature estimates, we prove two rigidity results for gradient shrinkers. More precisely, setting $C(n)=((n-2)$ $/(\sqrt{n(n-1)}))+\left(\left(n^{2}-n-4\right) /(2 \sqrt{(n-2)(n-1) n(n+1)})\right)$, which is defined in Lemma 10, we have the following Theorem 2.

Theorem 2. Assume that $\left(M^{n}, g, f\right)$ is a complete gradient shrinker of dimension $n \geq 3$. If

$$
|\stackrel{R}{R} m|^{2} \leq \frac{1}{C^{2}(n)}\left(\lambda-\frac{n-2}{n(n-1)} R\right)^{2}+\frac{4|\stackrel{R}{R} c|^{2}}{n},
$$

then $\left(M^{n}, g\right)$ is isometric to $\mathbb{R}^{n}$ or a finite quotient of $\mathbb{S}^{n}$. Moreover, when the pinching condition in the right hand of (6) is weakened to

$$
|\stackrel{R}{R} m|^{2}<\frac{2(n-1)}{n-2}\left(\lambda-\frac{n-2}{n(n-1)} R\right)^{2}+\frac{4|\stackrel{R}{R} c|^{2}}{n},
$$

then $\left(M^{n}, g\right)$ is Einstein

Remark 3. When

$$
|\stackrel{\circ}{R} m| \leq \frac{1}{C(n)}\left(\lambda-\frac{n-2}{n(n-1)} R\right),
$$

by equation (6) and Theorem 2, we see that $\left(M^{n}, g\right)$ is isometric to $\mathbb{R}^{n}$ or a finite quotient of $\mathbb{S}^{n}$. Therefore, Theorem 2 can be seen as a generalization of Theorem 1 .
Theorem 4. Let $\left(M^{n}, g, f\right)$ be a complete gradient shrinker of dimension $n \geq 3$ with nonnegative Ricci curvature. If

$$
|\stackrel{\circ}{R} m| \leq \frac{1}{C(n)}\left(\frac{R}{n(n-1)}+\frac{|\stackrel{\circ}{R} c|^{2}}{R}\right),
$$

then $\left(M^{n}, g\right)$ is isometric to $\mathbb{R}^{n}$ or a finite quotient of $\mathbb{S}^{n}$.

Remark 5. As is shown in the proof, the condition of nonnegative Ricci curvature in Theorem 4 can be relaxed to that $|R c| \leq c R^{1+\alpha}$ for some constants $c>0$ and $\alpha \geq 0$ satisfying $c R^{\alpha} \geq \sqrt{(n-2) /(n(n-1))}$.

Remark 6. Since any three-dimensional gradient shrinker must have nonnegative sectional curvature (cf. Corollary 2.4 of [13]), we see that the condition on Ricci curvature in Theorem 4 is not needed.

\section{Preliminaries of Curvature Estimates}

Let $\left(M^{n}, g\right)$ be a connected Riemannian manifold of dimension $n \geq 3$. In local coordinates, denoting by $R_{i j k l}, W_{i j k l}$, and $R^{\circ}{ }_{j k}=R_{j k}-(R / n) g_{j k}$ the components of the curvature tensor $R m$, the Weyl tensor $W$, and the traceless Ricci tensor $R^{\circ} c$, respectively, we have the well-known orthogonal decomposition of $\mathrm{Rm}$ (see e.g., [14]).

$$
\begin{aligned}
R_{i j k l}= & W_{i j k l}+\frac{R}{n(n-1)}\left(g_{i l} g_{j k}-g_{i k} g_{j l}\right) \\
& +\frac{1}{n-2}\left(\stackrel{\circ}{R}_{i l} g_{j k}+\stackrel{\circ}{R}_{j k} g_{i l}-\stackrel{\circ}{R}_{i k} g_{j l}-\stackrel{\circ}{R}_{j l} g_{i k}\right) .
\end{aligned}
$$

Correspondingly, the soliton equation (2) is rewritten as

$$
R_{i j}+\nabla_{i} \nabla_{j} f=\lambda g_{i j}
$$

Taking the trace in equation (11) gives

$$
R+\Delta f=n \lambda .
$$

Writing $R^{\circ} m=\left\{R_{i j k l}^{\circ}\right\}=\left\{R_{i j k l}-(R / n(n-1))\left(g_{i l} g_{j k}-\right.\right.$ $\left.\left.g_{i k} g_{j l}\right)\right\}$ and using the properties of $R m$, one can easily derive the following equalities:

$$
g^{i l} \stackrel{\circ}{R}_{i j k l}=\stackrel{\circ}{R}_{j k}
$$

$$
\begin{gathered}
\stackrel{\circ}{R}_{i j k l}+\stackrel{\circ}{R}_{i l j k}+\stackrel{\circ}{R}_{i k l j}=0, \\
\stackrel{\circ}{R}_{i j k l}=\stackrel{\circ}{R}_{k l i j}=-\stackrel{\circ}{R}_{j i k l}=-\stackrel{\circ}{R}_{i j l k}, \\
|\stackrel{\circ}{R} m|^{2}=|W|^{2}+\frac{4}{n-2}|\stackrel{\circ}{R} c|^{2},
\end{gathered}
$$


where the norm of a $(0,4)$-type tensor $T$ is defined by

$$
|T|^{2}=\left|T_{i j k l}\right|^{2}=g^{i m} g^{j n} g^{k s} g^{l t} T_{i j k l} T_{m n s t} \triangleq T^{i j k l} T_{i j k l}
$$

Here and subsequently, the notations $u_{*}={ }^{\Delta} \inf _{M^{n}} u$ as well as $u^{*}={ }^{\Delta} \sup _{M^{n}} u$ for a function $u$ on $M^{n}$ and Einstein summation convention are always adopted.

Recall the $f$-Laplacian $\Delta_{f}$, which is sometimes called the drifted Laplacian or Witten-Laplacian and is defined on a function $u \in \operatorname{Lip}_{\text {loc }}\left(M^{n}\right)$ by

$$
\Delta_{f} u=\Delta u-g(\nabla f, \nabla u)=e^{f} \operatorname{div}\left(e^{-f} \nabla u\right)
$$

in the weak sense, which is a self-adjoint operator on the space of square integrable functions on $\left(M^{n}, g, f\right)$ with respect to weighted volume form $\mathrm{e}^{-f} \mathrm{~d} V_{g}$. That is,

$$
\int_{M^{n}} \nabla \varphi \cdot \nabla \psi \mathrm{e}^{-f} \mathrm{~d} V_{g}=-\int_{M^{n}}\left(\Delta_{f} \varphi\right) \psi \mathrm{e}^{-f} \mathrm{~d} V_{g},
$$

for any $\varphi, \psi \in C_{0}^{\infty}\left(M^{n}\right)$, where $d V_{g}$ is the volume element induced by the metric $g$.

First of all, we will compute the $f$-Laplacian of the norm square of $R^{\circ} m$, by which we will establish the key estimate for any gradient Ricci soliton of dimension $n \geq 3$ in Lemma 10 . We start from Lemma 7.

Lemma 7. For any gradient Ricci soliton of dimension $n \geq 3$, we have

$$
\begin{aligned}
& 2 \stackrel{\circ i j k l}{R} \nabla_{l} \nabla^{p} R_{i j k p}-\frac{1}{2} \nabla|\stackrel{\circ}{R} m|^{2} \cdot \nabla f=2 \lambda|\stackrel{\circ}{R} m|^{2}-2 \stackrel{\circ}{R} \stackrel{i j k l}{R_{i j k}} \stackrel{p \circ}{R_{l p}} \\
& -\frac{2 R}{n}|\stackrel{\circ}{R} m|^{2}-\frac{4 R}{n(n-1)}|\stackrel{R}{n} c|^{2} \text {. }
\end{aligned}
$$

Proof. For convenience, we set

$$
\begin{aligned}
A & =2 \stackrel{\circ i j k l}{R} \nabla_{l} \nabla^{p} R_{i j k p}-\frac{1}{2} \nabla|\stackrel{\circ}{R} m|^{2} \cdot \nabla f \\
& =2 R^{\circ i j k l} \nabla_{l} \nabla^{p} R_{i j k p}-\stackrel{\circ i j k l}{R}\left(\nabla_{p} R_{i j k l}\right) \nabla^{p} f .
\end{aligned}
$$

On the one hand, by the second Bianchi identity, we get

$$
\begin{aligned}
& -\stackrel{\circ}{R}^{i j k l}\left(\nabla_{p} R_{i j k l}\right) \nabla^{p} f=-\stackrel{\circ}{R}^{i j k l}\left(\nabla_{l} R_{i j k p}-\nabla_{k} R_{i j l p}\right) \nabla^{p} f \\
& =-2 R^{\circ i j k l}\left(\nabla_{l} R_{i j k p}\right) \nabla^{p} f \text {. }
\end{aligned}
$$

On the other hand, by the Ricci identity and the equation (11), we deduce that

$$
\begin{aligned}
2 R^{\circ i j k l} \nabla_{l} \nabla^{p} R_{i j k p} & =2 R^{i j k l} \nabla_{l}\left(\nabla_{i} R_{j k}-\nabla_{j} R_{i k}\right) \\
& =2 R^{i j k l} \nabla_{l}\left(\nabla_{j} \nabla_{i} \nabla_{k} f-\nabla_{i} \nabla_{j} \nabla_{k} f\right) \\
& =2 R^{i j k l}\left(\nabla_{l} R_{i j k p}\right) \nabla^{p} f+2 \stackrel{\circ}{R}^{i j k l} R_{i j k p}\left(\lambda g_{l}^{p}-R_{l}^{p}\right) .
\end{aligned}
$$

Combining the facts $R_{i j k p}=R_{i j k p}^{\circ}+(R / n(n-1))\left(g_{i p} g_{j k}\right.$ $\left.-g_{i k} g_{j p}\right)$ and $R_{i j}=R_{i j}^{\circ}+(R / n) g_{i j}$ with (23) yields

$$
\begin{aligned}
& A=2 \lambda|\stackrel{\circ}{R} m|^{2}-2 \stackrel{\circ}{R}^{i j k l}\left({\stackrel{\circ}{R_{i j k}}}^{p}+\frac{R}{n(n-1)}\left(g_{i}^{p} g_{j k}-g_{i k} g_{j}^{p}\right)\right)\left(\stackrel{\circ}{R}_{l p}+\frac{R}{n} g_{l p}\right) .
\end{aligned}
$$

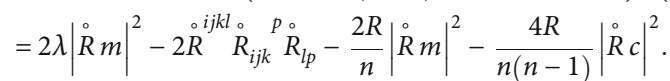

Our next step is to compute the Laplacian of $\left|R^{\circ} m\right|^{2}$ for all Riemannian manifolds.

Lemma 8. Let $\left(M^{n}, g\right)$ be an $n$-dimensional $(n \geq 3)$ complete Riemannian manifold. Then,

$$
\begin{aligned}
& \frac{1}{2} \Delta\left|\stackrel{\circ}{R}_{i j k l}\right|^{2}=|\nabla \stackrel{\circ}{R} m|^{2}-2 \stackrel{\circ}{R}^{i j k l}\left(2 \stackrel{\circ}{2}{ }_{i l} \stackrel{\circ}{R}_{h j k p}-\frac{1}{2} \stackrel{\circ}{R}_{i j h p} \stackrel{\circ}{R}_{k l}{ }_{k l}-\stackrel{\circ}{R}_{i j k} \stackrel{\circ}{\circ}_{l h}\right) \\
& +2 \stackrel{\circ}{R}^{i j k l} \nabla_{l} \nabla^{p} R_{i j k p}+\frac{2 R}{n}|\stackrel{\circ}{R} m|^{2}-\frac{4 R}{n(n-1)}|\stackrel{\circ}{R} c|^{2} .
\end{aligned}
$$

Proof. By the definitions of $R^{\circ} m,\left|R^{\circ} m\right|^{2}$, and (13), we have

$$
\frac{1}{2} \Delta\left|\stackrel{\circ}{R}_{i j k l}\right|^{2}=|\nabla \stackrel{\circ}{R} m|^{2}+\stackrel{\circ i j k l}{R} \nabla^{p} \nabla_{p} \stackrel{\circ}{R}_{i j k l}=|\nabla \stackrel{\circ}{R} m|^{2}+\stackrel{\circ i j k l}{R} \nabla^{p} \nabla_{p} R_{i j k l} .
$$

Employing Bianchi's second identity, we obtain

$$
\begin{aligned}
& \stackrel{\circ i j k l}{R} \nabla^{p} \nabla_{p} R_{i j k l}=\stackrel{\circ i j k l}{R} \nabla^{p}\left(\nabla_{l} R_{i j k p}-\nabla_{k} R_{i j l p}\right)
\end{aligned}
$$

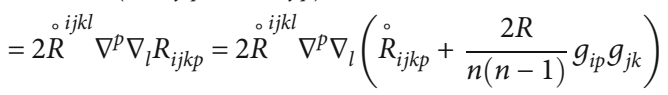

$$
\begin{aligned}
& =2 \stackrel{\circ}{R}^{i j k l} \nabla^{p} \nabla_{l} \stackrel{\circ}{R}_{i j k p}+\frac{4 \stackrel{\circ p l}{R}}{n(n-1)} \nabla_{p} \nabla_{l} R,
\end{aligned}
$$

which together with (26) implies

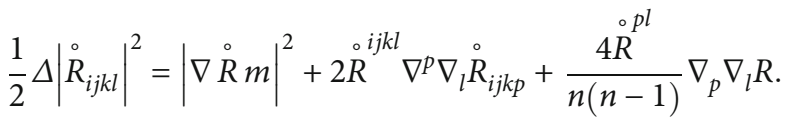


Making use of the Ricci identity and (13), we have

$$
\begin{aligned}
& 2 \stackrel{\circ}{R}^{i j k l} \nabla^{p} \nabla_{l} \stackrel{\circ}{R}_{i j k p}=2 \stackrel{\circ}{R}^{i j k l}\left(\nabla_{l} \nabla^{p} \stackrel{\circ}{R}_{i j k p}-R^{p}{ }_{l i}^{h} \stackrel{\circ}{R}_{h j k p}\right. \\
& \left.+R_{l j}^{p}{ }_{l j}^{h} R_{h i k p}-R^{p}{ }_{l k}{ }^{h} R_{i j h p}-R_{l p}^{p}{ }_{l}{ }^{\circ} R_{i j k h}\right)
\end{aligned}
$$

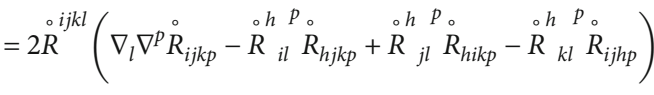

$$
\begin{aligned}
& +2 R \stackrel{\circ i j k l}{R} R_{i j k}^{h}\left(\stackrel{\circ}{R}_{l h}+\frac{R}{n} g_{l h}\right)-\frac{2 R}{n(n-1)} \stackrel{\circ}{R}^{i j k l} \\
& \cdot\left(\stackrel{\circ}{R}_{h j k p}\left(g^{h p} g_{i l}-g_{l}^{h} g_{i}^{p}\right)-\stackrel{\circ}{R}_{h i k p}\left(g^{h p} g_{j l}-g_{l}^{h} g_{j}^{p}\right)\right. \\
& \left.+\stackrel{\circ}{R}_{i j h p}\left(g^{h p} g_{k l}-g_{l}^{h} g_{k}^{p}\right)\right)
\end{aligned}
$$

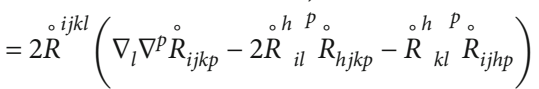

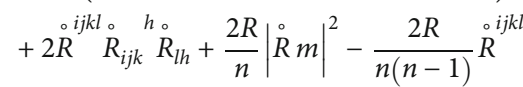

$$
\begin{aligned}
& \cdot\left(-\stackrel{\circ}{R}_{l j k i}-\stackrel{\circ}{R}_{i l k j}-\stackrel{\circ}{R}_{i j l k}+\stackrel{\circ}{R}_{j k} g_{l i}-\stackrel{\circ}{R}_{i k} g_{l j}\right) .
\end{aligned}
$$

Combining (13) and (14) with (29), we get

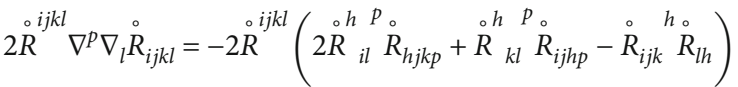

$$
\begin{aligned}
& +2 R^{\circ i j k l} \nabla_{l} \nabla^{p} \stackrel{\circ}{i j k p}+\frac{2 R}{n}|\stackrel{\circ}{R} m|^{2}-\frac{4 R}{n(n-1)}|\stackrel{\circ}{R} c|^{2},
\end{aligned}
$$

where

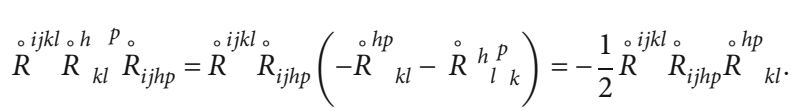

Substituting (30) and (31) into (28), we obtain

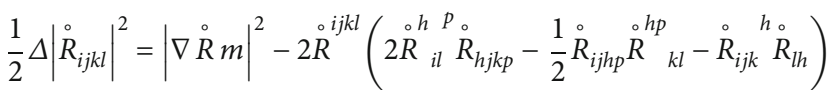

$$
\begin{aligned}
& +2 \stackrel{\circ i j k l}{R} \nabla_{l} \nabla^{p} R_{i j k p}+\frac{2 R}{n}|\stackrel{\circ}{R} m|^{2}-\frac{4 R}{n(n-1)}|\stackrel{\circ}{R} c|^{2},
\end{aligned}
$$

where the formula

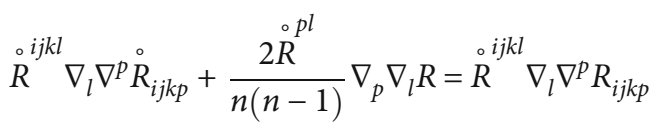

is used in (32).

By Lemmas 7 and 8 and the fact that

$$
\frac{1}{2} \Delta_{f}\left|\stackrel{\circ}{R}_{i j k l}\right|^{2}=\frac{1}{2} \Delta\left|\stackrel{\circ}{R}_{i j k l}\right|^{2}-\stackrel{\circ}{R}\left(\nabla_{p} R_{i j k l}\right) \nabla^{p} f,
$$

we now arrive at the $f$-Laplacian formula of $\left|R^{\circ} m\right|^{2}$ for all gradient Ricci solitons.

Lemma 9. Let $\left(M^{n}, g, f\right)$ be an $n$-dimensional $(n \geq 3)$ gradient Ricci soliton. Then,

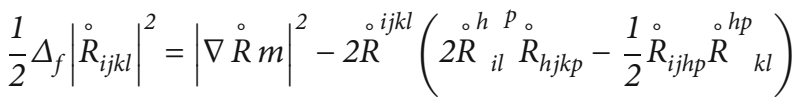

$$
\begin{aligned}
& +2 \lambda|\stackrel{\circ}{R} m|^{2}-\frac{8 R}{n(n-1)}|\stackrel{\circ}{R} c|^{2} \text {. }
\end{aligned}
$$

Consequently, we conclude from (35) and (16) that

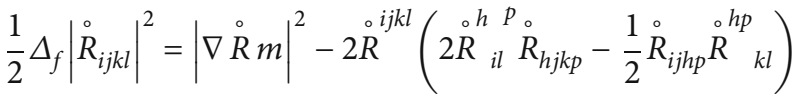

$$
\begin{aligned}
& +2 \lambda|\stackrel{\circ}{R} m|^{2}-\frac{2(n-2) R}{n(n-1)}|\stackrel{\circ}{R} m|^{2} \\
& +\frac{2(n-2) R}{n(n-1)}|W|^{2} \text {, }
\end{aligned}
$$

for any gradient Ricci soliton.

Utilizing the inequalities proved by Li and Zhao [15] and Huisken [16] (see also [17]), we have

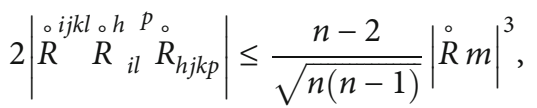

$$
\left|\stackrel{\circ i j k l}{R} \stackrel{\circ}{R_{i j h p}} \stackrel{\circ}{h p}_{k l}\right| \leq \frac{n^{2}-n-4}{\sqrt{(n-2)(n-1) n(n+1)}}|\stackrel{\circ}{R} m|^{3} .
$$

Combining (37) and (38) with (36) gives

Lemma 10. Let $\left(M^{n}, g, f\right)$ be an $n$-dimensional $(n \geq 3)$ gradient Ricci soliton. Then,

$$
\begin{aligned}
\frac{1}{2} \Delta_{f}|\stackrel{\circ}{R} m|^{2} \geq & |\nabla \stackrel{\circ}{R} m|^{2} \\
& -2\left(\frac{n-2}{\sqrt{n(n-1)}}+\frac{n^{2}-n-4}{2 \sqrt{(n-2)(n-1) n(n+1)}}\right)|\stackrel{\circ}{R} m|^{3} \\
& +2 \lambda|\stackrel{\circ}{R} m|^{2}-\frac{2(n-2) R}{n(n-1)}|\stackrel{\circ}{R} m|^{2} \\
& +\frac{2(n-2) R}{n(n-1)}|W|^{2} \triangleq|\nabla \stackrel{\circ}{R} m|^{2}-2 C(n)|\stackrel{\circ}{R} m|^{3} \\
& +2\left(\lambda-\frac{(n-2) R}{n(n-1)}\right)|\stackrel{\circ}{R} m|^{2}+\frac{2(n-2) R}{n(n-1)}|W|^{2} .
\end{aligned}
$$

Remark 11. Inequality (39) can also be derived by setting $X=\nabla f$ in Lemma 2.5 of [12]; here, we give its proof for the sake of completeness. 
Correspondingly, the $f$-Laplacian of $\left|R^{\circ} c\right|^{2}$ is (see e.g. Lemma 2.1 of [18])

Lemma 12. Let $\left(M^{n}, g, f\right)$ be a gradient Ricci soliton of dimension $n \geq 3$. Then,

$$
\begin{aligned}
& \frac{1}{2} \Delta_{f}|\stackrel{\circ}{R} c|^{2}=|\nabla \stackrel{\circ}{R} c|^{2}+2\left(\lambda-\frac{(n-2) R}{n(n-1)}\right)|\stackrel{\circ}{R} c|^{2}
\end{aligned}
$$

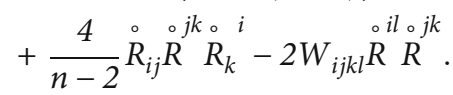

Employ the following curvature inequality.

Lemma 13. (Proposition 2.1 of [19]). Let $\left(M^{n}, g\right)$ be an $n$ -dimensional $(n \geq 3)$ Riemannian manifold. Then,

$$
\begin{aligned}
& \left|-W_{i j k l} \stackrel{\circ i l \circ j k}{R} R+\frac{2}{n-2} \stackrel{\circ}{R} \stackrel{\circ j}{R} \stackrel{p}{i}_{R} R_{p j}\right| \\
& \leq \sqrt{\frac{n-2}{2(n-1)}}\left(|W|^{2}+\frac{8}{n(n-2)}|\stackrel{\circ}{R} c|^{2}\right)^{\frac{1}{2}}|\stackrel{\circ}{R} c|^{2} \text {. }
\end{aligned}
$$

We deduce from Lemma 12 the following.

Lemma 14. Let $\left(M^{n}, g\right)$ be an $n$-dimensional $(n \geq 3)$ Riemannian manifold. Then,

$$
\begin{gathered}
\frac{1}{2} \Delta_{f}|\stackrel{\circ}{R} c|^{2} \geq|\nabla \stackrel{\circ}{R} c|^{2}+2\left(\lambda-\frac{(n-2) R}{n(n-1)}-\sqrt{\frac{n-2}{2(n-1)}}\right. \\
\left.\cdot\left(|W|^{2}+\frac{8}{n(n-2)}|\stackrel{R}{ } c|^{2}\right)^{\frac{1}{2}}\right)|\stackrel{\circ}{R} c|^{2} .
\end{gathered}
$$

Since the scalar curvature of nonflat Ricci shrinker is positive, by Proposition 2.7 of [12], we get the following curvature inequality.

Lemma 15. Let $\left(M^{n}, g, f\right)$ be an $n$-dimensional $(n \geq 3)$ complete nonflat gradient shrinker. Then,

$$
\begin{aligned}
\Delta_{f-2 \nabla \log R}\left(\frac{|\stackrel{\circ}{R} m|^{2}}{R^{2}}\right) \geq & \frac{2}{R^{3}}\left(\sqrt{R}|\nabla \stackrel{\circ}{R} m|-\frac{|\stackrel{\circ}{R} m||\nabla R|}{\sqrt{R}}\right)^{2} \\
& +\frac{4(n-2)|W|^{2}}{n(n-1) R}+\frac{4|\stackrel{\circ}{R} m|^{2}}{R^{3}} \\
& \cdot\left(\frac{R^{2}}{n(n-1)}+|\stackrel{\circ}{R} c|^{2}-C(n) R|\stackrel{\circ}{R} m|\right) .
\end{aligned}
$$

For the sake of the proofs of our main theorems, we recall the following results due to Catino [10], Pigola et al. [20], and Petersen and Wylie [8] (see also [4]).

Lemma 16 (Proposition 1 of [10]). Let $\left(M^{n}, g, f\right)$ be a complete gradient nonflat shrinker of dimension $n \geq 3$. Then,

$$
\begin{aligned}
\Delta_{f-2 \nabla \log R}\left(\frac{|\stackrel{\circ}{R} c|^{2}}{R^{2}}\right) \geq & \frac{2}{R^{4}}\left|R \nabla_{j} R_{i k}-\nabla_{j} R R_{i k}\right|^{2} \\
& +\frac{4}{R^{3}}\left[|\stackrel{\circ}{R}|^{2}\left(|\stackrel{R}{R} c|-\frac{R}{\sqrt{n(n-1)}}\right)^{2}\right. \\
& \left.-R W_{i j k l} \stackrel{\circ i l \circ j k}{R}\right] .
\end{aligned}
$$

Lemma 17 (Theorem 3 of [20]). Let $\left(M^{n}, g, f\right)$ be a complete gradient shrinker of dimension $n \geq 3$. Then, $0 \leq R_{*} \leq n \lambda$. Moreover, $R_{*}<n \lambda$ unless $(M, g)$ is Einstein and the soliton is trivial, and $R_{*}>0$ unless $R \equiv 0$ and $(M, g)$ is isometric to $\mathbb{R}^{n}$.

Lemma 18 (Theorem 22 of [20]). Any complete gradient shrinker $\left(M^{n}, g, f\right)$ is $f$-parabolic, namely, every solution of $\Delta_{f} u \geq 0$ satisfying $u^{*}<+\infty$ must be a constant.

Lemma 19 (Lemma 4.2 of [8]). Assume that $\left(M^{n}, g\right)$ is an $n$-dimensional manifold with finite $w$-volume, i.e., $\int_{M} e^{-w} d$ $V_{g}<+\infty$. If a smooth function $u \in L^{2}\left(e^{-w} d V_{g}\right)$ is bounded below such that $\Delta_{w} u \geq 0$, then $u$ is a constant.

\section{Proofs of Main Theorems}

We are now in a position to give the proofs of our main theorems.

Proof of Theorem 2. Using (16), we see that pinching conditions (6) and (7) in Theorem 2 are equivalent to the following inequality, respectively:

$$
\begin{aligned}
& |W|^{2}+\frac{8}{n(n-2)}|\stackrel{\circ}{R} c|^{2} \leq \frac{1}{C^{2}(n)}\left(\lambda-\frac{n-2}{n(n-1)} R\right)^{2}, \\
& |W|^{2}+\frac{8}{n(n-2)}|\stackrel{\circ}{R} c|^{2}<\frac{2(n-1)}{n-2}\left(\lambda-\frac{n-2}{n(n-1)} R\right)^{2} .
\end{aligned}
$$


By Lemma 14 and (46), we have

$$
\begin{aligned}
\frac{1}{2} \Delta_{f}|\stackrel{R}{R} c|^{2} \geq & |\nabla \stackrel{\circ}{R} c|^{2}+2\left(\lambda-\frac{(n-2) R}{n(n-1)}-\sqrt{\frac{n-2}{2(n-1)}}\right. \\
& \left.\cdot\left(|W|^{2}+\frac{8}{n(n-2)}|\stackrel{\circ}{R} c|^{2}\right)^{1 / 2}\right)|\stackrel{\circ}{R} c|^{2} \\
\geq & 2\left(\lambda-\frac{(n-2) R}{n(n-1)}-\sqrt{\frac{n-2}{2(n-1)}}\right. \\
& \left.\cdot\left(|W|^{2}+\frac{8}{n(n-2)}|\stackrel{\circ}{R} c|^{2}\right)^{1 / 2}\right)|\stackrel{\circ}{R} c|^{2} \geq 0,
\end{aligned}
$$

which along with Lemma 18 and (46) yields $\left|R^{\circ} c\right|=0$ and therefore $\left(M^{n}, g\right)$ is Einstein.

On the other hand, if (45) holds, it is easily seen that (46) also holds. Indeed, when $n=3$, clearly $1 / C^{2}(3)=2 / 3<4$. When $n \geq 4$, we see from the fact

$C(n)=\frac{n-2}{\sqrt{n(n-1)}}+\frac{n^{2}-n-4}{2 \sqrt{(n-2)(n-1) n(n+1)}}>\frac{n-2}{\sqrt{n(n-1)}}$

that

$$
\frac{1}{C^{2}(n)}<\frac{n(n-1)}{(n-2)^{2}} \leq \frac{2(n-1)}{n-2}
$$

It follows from Lemma 18 and (45) that $\left|R^{\circ} c\right|=0$, which together with (16) implies

$$
|\stackrel{\circ}{R} m|^{2}=|W|^{2} \leq \frac{1}{C^{2}(n)}\left(\lambda-\frac{n-2}{n(n-1)} R\right)^{2} .
$$

By Lemma 10 and (50) we know that

$$
\begin{aligned}
\frac{1}{2} \Delta_{f}|\stackrel{\circ}{R} m|^{2} \geq & |\nabla \stackrel{\circ}{R} m|^{2}+2\left(\lambda-\frac{(n-2) R}{n(n-1)}-C(n)|\stackrel{\circ}{R} m|\right)|\stackrel{\circ}{R} m|^{2} \\
& +\frac{2(n-2) R}{n(n-1)}|W|^{2} \\
\geq & 2\left(\lambda-\frac{(n-2) R}{n(n-1)}-C(n)|\stackrel{\circ}{R} m|\right)|\stackrel{\circ}{R} m|^{2} \geq 0,
\end{aligned}
$$

where the fact that $R \geq 0$ for shrinking solitons (see Lemma 17 or Corollary 2.5 of [13]) is used in the second inequality in (51). It follows from Lemma 18 and (50) that $\left|R^{\circ} \mathrm{m}\right|$ is a constant and therefore all equalities in (51) hold.

If there exists $x_{0} \in M^{n}$ such $R\left(x_{0}\right)=0$, then, we see from Lemma 17 that $M^{n}$ is isometric to $\mathbb{R}^{n}$.

Otherwise, the facts $R>0,\left|R^{\circ} c\right|=0$ and the equalities of (51) imply that $R^{\circ} m=W=0$. Hence, we know that $\left(M^{n}, g\right)$ has constant sectional curvature when $R>0$; it follows from the Myers theorem and the condition $R>0$ that $\left(M^{n}, g\right)$ is compact and therefore is a finite quotient of $\mathbb{S}^{n}$.

Proof of Theorem 4. It is well known that $R \geq 0$ for shrinking solitons. When $R$ achieves its infimum 0 , Lemma 17 says that $\left(M^{n}, g, f\right)$ is flat and therefore is isometric to $\mathbb{R}^{n}$.

In the rest of the proofs of Theorem 4, we assume that $R>0$. By (43) and (9), we see that

$$
\begin{aligned}
\Delta_{f-2 \nabla \log R}\left(\frac{|\stackrel{\circ}{R} m|^{2}}{R^{2}}\right) \geq & \frac{2}{R^{3}}\left(\sqrt{R}|\nabla \stackrel{\circ}{R} m|-\frac{|\stackrel{\circ}{R} m||\nabla R|}{\sqrt{R}}\right)^{2} \\
& +\frac{4(n-2)|W|^{2}}{n(n-1) R}+\frac{4|\stackrel{\circ}{R} m|^{2}}{R^{3}} \\
& \cdot\left(\frac{R^{2}}{n(n-1)}+|\stackrel{\circ}{R} c|^{2}-C(n) R|\stackrel{\circ}{R} m|\right) \\
\geq & \frac{4|\stackrel{\circ}{R} m|^{2}}{R^{3}}\left(\frac{R^{2}}{n(n-1)}+|\stackrel{\circ}{R} c|^{2}-C(n) R|\stackrel{\circ}{R} m|\right) \\
\geq & 0 .
\end{aligned}
$$

Set $w=f-\nabla \log \left(R^{2}\right)$ and $u=\left(\left|R^{\circ} m\right|^{2}\right) / R^{2}$. In order to apply Lemma 19 to (52), we need to verify

$$
\begin{gathered}
\int_{M^{n}} \mathrm{e}^{-w} \mathrm{~d} V_{g}=\int_{M^{n}} R^{2} \mathrm{e}^{-f} \mathrm{~d} V_{g}<+\infty, \\
\int_{M^{n}} \frac{|\stackrel{\circ}{R} m|^{4}}{R^{4}} \mathrm{e}^{-w} \mathrm{~d} V_{g}=\int_{M^{n}} \frac{|\stackrel{\circ}{R} m|^{4}}{R^{2}} \mathrm{e}^{-f} \mathrm{~d} V_{g}<+\infty .
\end{gathered}
$$

In fact, (53) follows from the result that $R \in L^{p}\left(\mathrm{e}^{-f} \mathrm{~d} V_{g}\right)$ for $1 \leq p<+\infty$ (see e.g., [10]) for all gradient shrinkers.

Under the assumption that $\left(M^{n}, g, f\right)$ has nonnegative Ricci curvature and (9), we get $|R c| \leq R$. Thus,

$$
\begin{aligned}
& |\stackrel{\circ}{R} m| \leq \frac{1}{C(n)}\left(R-\frac{(n-2) R}{n(n-1)}\right)=\frac{n^{2}-2 n+2}{n(n-1) C(n)} R, \\
& \frac{|\stackrel{\circ}{R} m|^{4}}{R^{2}} \leq\left(\frac{n^{2}-2 n+2}{n(n-1) C(n)}\right)^{4} R^{2} \in L\left(\mathrm{e}^{-f} \mathrm{~d} V_{g}\right) .
\end{aligned}
$$

Furthermore, as observed in Remark 5, if we relax the condition of negative Ricci curvature to that $|R c| \leq c R^{1+\alpha}$ for some constants $c>0$ and $\alpha \geq 0$ satisfying $c R^{\alpha} \geq$ $\sqrt{(n-2) /(n(n-1))}$, then

$$
|\stackrel{\circ}{R} m| \leq \frac{R}{C(n)}\left(c^{2} R^{2 \alpha}-\frac{n-2}{n(n-1)}\right)
$$

It follows from the result $R \in L^{p}\left(\mathrm{e}^{-f} \mathrm{~d} V_{g}\right)$ for $1 \leq p<+$ $\infty$ that 


$$
\frac{|\stackrel{\circ}{R} m|^{4}}{R^{2}} \in L\left(\mathrm{e}^{-f} \mathrm{~d} V_{g}\right)
$$

These together with (52) and Lemma 19 yield $R^{\circ} m=0$ or $W=0$ and $\left|R^{\circ} m\right|=1 / C(n)\left((R /(n(n-1)))+\left(\left|R^{\circ} c\right|^{2} / R\right)\right)$.

What we need to prove now is that $R^{\circ} c=0$ in the latter case. In fact, by (16), the facts $W=0$ and $\left|R^{\circ} m\right|=$ $1 / C(n)\left((R /(n(n-1)))+\left(\left|R^{\circ} c\right|^{2} / R\right)\right)$, we derive

$$
|\stackrel{\circ}{R} c|=\frac{\sqrt{n-2}}{2}|\stackrel{\circ}{R} m|=\frac{\sqrt{n-2}}{2 C(n)}\left(\frac{R}{n(n-1)}+\frac{|\stackrel{\circ}{R} c|^{2}}{R}\right) .
$$

It is easy to check from the definition of $C(n)$ that the different two solutions of equation (58) satisfy

$$
|\stackrel{R}{\mid} c|_{1}=\frac{C(n) R}{\sqrt{n-2}}+R \sqrt{\frac{C^{2}(n)}{n-2}-\frac{1}{n(n-1)}}>\frac{\sqrt{n-2}}{\sqrt{n(n-1)}} R,
$$

$$
\begin{aligned}
|\stackrel{R}{R}|_{2} & =\frac{(1 /(n(n-1))) R^{2}}{(C(n) R / \sqrt{n-2})+R \sqrt{\left(C^{2}(n) / n-2\right)-(1 /(n(n-1)))}} \\
& <\frac{R}{\sqrt{(n-2)(n-1) n}} .
\end{aligned}
$$

Combining (59) and (60) and the fact $W=0$ with Lemma 16 gives

$$
\begin{aligned}
\Delta_{f-2 \nabla \log R}\left(\frac{|\stackrel{\circ}{R} c|^{2}}{R^{2}}\right) & \geq \frac{2}{R^{4}}\left|R \nabla_{j} R_{i k}-\nabla_{j} R R_{i k}\right|^{2}+\frac{4|\stackrel{\circ}{R} c|^{2}}{R^{3}} \\
& \cdot\left(|\stackrel{R}{R} c|-\frac{R}{\sqrt{n(n-1)}}\right)^{2} \\
\geq & \frac{4|\stackrel{R}{R}|^{2}}{R^{3}}\left(|\stackrel{\circ}{R} c|-\frac{R}{\sqrt{n(n-1)}}\right)^{2} \\
\geq & 0 .
\end{aligned}
$$

By a similar argument, we conclude from Lemma 19 and the assumption on the Ricci curvature that $\left|R^{\circ} \mathrm{c}\right|^{2} / R$ is a constant and $R^{\circ} c=0$ since $\left|R^{\circ} c\right|_{1,2} \neq R / \sqrt{n(n-1)}$. This concludes the proof of Theorem 4 .

\section{Data Availability}

No data were used to support this study.

\section{Conflicts of Interest}

The authors declare that they have no conflicts of interest.

\section{Acknowledgments}

The first author would like to express his sincere gratitude to Prof. Xi Zhang and University of Science and Technology of China for their support. The research was partially supported by the project "Analysis and Geometry on Bundle" of the Ministry of Science and Technology of the People's Republic of China (No. SQ2020YFA070080) and NSF of China (Nos. 11625106, 11801535, 11721101, and 11801011). The authors were partially supported by the NSF of Anhui Provincial Education Department (Nos. KJ2018A0330, gxgnfx2018017, 2019mooc205, and 2019MSZGS01).

\section{References}

[1] R. Hamilton, "The formation of singularities in the Ricci flow," Surveys in differential geometry, vol. 2, pp. 7-136, 1995.

[2] T. Ivey, "Ricci solitons on compact three-manifolds," Differential Geometry and its Applications, vol. 3, no. 4, pp. 301-307, 1993.

[3] G. Perelman, "Ricci flow with surgery on three-manifolds," 2003, https://arxiv.org/abs/math/0303109.

[4] A. Naber, "Noncompact shrinking four solitons with nonnegative curvature," Journal fur die Reine und Angewandte Mathematik, vol. 645, pp. 125-153, 2010.

[5] L. Ni and N. Wallach, "On a classification of gradient shrinking solitons," Mathematical Research Letters, vol. 15, no. 5, pp. 941-955, 2008.

[6] H. D. Cao, B. L. Chen, and X. P. Zhu, "Recent developments on Hamilton's Ricci flow," Surveys in diferential geometry, vol. 12, pp. 47-112, 2008.

[7] O. Munteanu and N. Sesum, "On gradient Ricci solitons," Journal of Geometric Analysis, vol. 23, no. 2, pp. 539-561, 2013.

[8] P. Petersen and W. Wylie, "On the classification of gradient Ricci solitons," Geometry and Topology, vol. 14, no. 4, pp. 2277-2300, 2010.

[9] Z. H. Zhang, "Gradient shrinking solitons with vanishing Weyl tensor," Pacific Journal of Mathematics, vol. 242, no. 1, pp. 189-200, 2009.

[10] G. Catino, "Complete gradient shrinking Ricci solitons with pinched curvature," Mathematische Annalen, vol. 355, no. 2, pp. 629-635, 2013.

[11] O. Munteanu and J. Wang, "Geometry of shrinking Ricci solitons," Compositio Mathematica, vol. 151, no. 12, pp. 22732300, 2015.

[12] Y. W. Chu, R. Huang, and W. W. Li, "Classification of complete generic shrinking Ricci solitons with pointwise pinched curvature," Differential Geometry and its Applications, vol. 54, pp. 385-396, 2017.

[13] B. L. Chen, "Strong uniqueness of the Ricci flow," Journal of Differential Geometry, vol. 82, no. 2, pp. 362-382, 2009.

[14] A. L. Besse, Einstein manifolds, Springer-Verlag, Berlin, 2008.

[15] A. M. Li and G. S. Zhao, "Isolation phenomena for Riemannian manifolds whose Ricci curvature tensor are parallel," Acta Mathematica Scientia. Series A, vol. 37, no. 1, pp. 19-24, 1994. 
[16] G. Huisken, "Ricci deformation of the metric on a Riemannian manifold," Journal of Differential Geometry, vol. 21, pp. 47-62, 1985.

[17] H. P. Fu and L. Q. Xiao, "Some $\mathrm{L}^{\mathrm{p}}$ rigidity results for complete manifolds with harmonic curvature," Potential Analysis, vol. 48 , no. 2, pp. 239-255, 2018.

[18] G. Catino, P. Mastrolia, D. D. Monticelli, and M. Rigoli, “Analytic and geometric properties of generic Ricci solitons," Transactions of the American Mathematical Society, vol. 368, no. 11, pp. 7533-7549, 2016.

[19] G. Catino, "Integral pinched shrinking Ricci solitons," Advances in Mathematics, vol. 303, pp. 279-294, 2016.

[20] S. Pigola, M. Rigoli, and A. G. Setti, "Remarks on non-compact gradient Ricci solitons," Mathematische Zeitschrift, vol. 268, no. 3-4, pp. 777-790, 2011. 